\title{
Características clínicas da leishmaniose tegumentar em crianças de 0 a 5 anos em uma área endêmica de Leishmania (Viannia) braziliensis
}

\author{
Clinical findings of tegumentary leishmaniasis in children under five years \\ of age in an endemic area of Leishmania (Viannia) braziliensis
}

\author{
Julia Ampuero $^{1}$, Vanize Macêdo ${ }^{1}$ e Philip Marsden ${ }^{\dagger}$
}

\begin{abstract}
RESUMO
Para caracterizar a clínica da leishmaniose tegumentar em crianças de 0 a 5 anos de idade, foram avaliadas, retrospectivamente, 4.464 fichas clínicas do Centro de Saúde de Corte de Pedra, Presidente Tancredo Neves, Bahia, Brasil, área endêmica de leishmaniose tegumentar americana, entre maio de 1987 e dezembro de 1995. Foram registrados neste período 4.275 casos novos de leishmaniose, dos quais, 491 (11,5\%) correspondiam a crianças de 0 a 5 anos. A razão entre gênero masculino e feminino nas crianças foi 1,1:1. A forma clínica predominante foi a cutânea (98\%) e as lesões ulceradas foram as mais freqüentes (99\%). A localização das lesões ocorreu, principalmente, acima da cintura ( $p<0,05)$, e 35,5\% apresentaram lesões múltiplas. A magnitude da doença em crianças, a freqüência semelhante observada em ambos os gêneros e a localização das lesões sugere a possibilidade de transmissão vetorial no domicilio ou peridomicîlio.
\end{abstract}

Palavras-chaves: Leishmaniose tegumentar. Características clínicas. Leishmania (Viannia) braziliensis.

\section{ABSTRACT}

A retrospective study was performed in a field clinic to characterize the clinical findings of tegumentary leishmaniasis in children from 0 to 5 years old. The clinical records of 4,464 patients were analyzed at the Primary Health Center of Corte de Pedra, Presidente Tancredo Neves, Bahia, Brazil, from May 1987 to December 1995. Four hundred and ninety one (11.8\%) children were identified among 4,275 new cases of tegumentary leishmaniasis registered at this Unit. The gender ratio (M:F) for children under six years was 1.1:1. Cutaneous lesions predominated (98\%), mainly skin ulcers (99\%) located above the waist $(p<0.05)$. Thirty five percent had multiple lesions. The observed magnitude of the disease in children, the similar proportion of cases in both genders and the location of the lesions suggest the possibility of peri or intradomiciliary transmission.

Key-words: Tegumentary leishmaniasis. Clinical findings. Leishmania (Viannia) braziliensis.

A leishmaniose tegumentar (LT) é uma doença de alta prevalência em muitas áreas tropicais e subtropicais do mundo. Em vários paises da América Latina constitui um grave problema de Saúde Pública pelo impacto negativo que representa nas esferas psicológica, sociocultural e econômica ${ }^{159}$.

No Brasil, todos os estados da Federação registraram casos de LT com uma frequiência constante a partir do ano 2000. Durante 2004, o Estado da Bahia, na região Nordeste, foi o segundo Estado depois do Maranhão, em relatar maior numero de casos. Aproximadamente, um $(26,2 \%)$ em cada quatro pacientes foi registrado na área de influência do Centro de Saúde de Corte de Pedra (CSCP) localizado na messoregião sul da Bahia.

Nesta região, foram descritas duas espécies de leishmania: L. (Viannia) braziliensis, responsável por mais de $95 \%$ dos casos, e L. (Leishmania) amazonensis, de ocorrência mais rara ${ }^{2324}$.

No Brasil, como em outros países da América, a maioria dos estudos clínico-epidemiológicos sobre leishmanioses têm sido dirigida aos grupos de jovens e adultos; poucos são os estudos realizados sobre leishmanioses em crianças ${ }^{4814212227}$. As publicações sobre este grupo etário referem-se quase sempre aos intervalos de idade correspondendo às faixas etárias 0 a 9,

\footnotetext{
1.Núcleo de Medicina Tropical da Universidade de Brasíilia, Brasília, DF, Brasil.

Endereço para correspondência: Dra. Julia Ampuero. Núcleo de Medicina Tropical/UnB. Campus Universitário. Asa Norte. Caixa Postal 4517, 70199-970 Brasília, DF. Brasil. Tel: $55613273-5008$, Fax: $55613273-2811$.

e-mail: ausangat@terra.com.pe, juliaampuero@unb.br

${ }^{\dagger}$ In memoriam

Recebido para publicação em 28/11/2004

Aceito em 30/8/2005
} 
10 a 19,0 a 10 anos $^{3161926}$, ou de 0 a 15 anos de idade ${ }^{11}$, perdendose os detalhes referentes aos grupos de idade mais baixos. São poucos os trabalhos que estratificaram, minuciosamente, os grupos etários $^{1425}$. 0 diagnóstico e tratamento precoces são importantes, para evitar a progressão da doença que poderá estigmatizar a criança pelo resto da vida.

0 objetivo do presente estudo foi caracterizar as formas de apresentação clínica da leishmaniose tegumentar em crianças de 0 a 5 anos na área de influência geográfica do CSCP, realizando comparações do comportamento da doença com indivíduos jovens e adultos, avaliados na mesma Unidade de Saúde, para contribuir ao diagnóstico precoce da enfermidade nesta faixa etária.

\section{PACIENTES E MÉTODOS}

O estudo foi desenvolvido no CSCP, centro de referência para os pacientes de leishmaniose na região, situado no distrito de Corte de Pedra, pertencente ao município de Presidente Tancredo Neves, localizado na messoregião sul da Bahia. Corte de Pedra está situado às margens da rodovia BR 101, à distância aproximada de $275 \mathrm{Km}$ de Salvador capital do Estado. O Núcleo de Medicina Tropical da Universidade de Brasília (UnB) e o Serviço de Imunologia da Universidade Federal da Bahia (UFBA) coordenam o atendimento dos pacientes, desenvolvendo nesta área ensaios clínicos e de controle da doença.

Foram diagnosticados como casos de LT aqueles pacientes com suspeita clínica e alguma prova de confirmação laboratorial (exame parasitológico ou diagnóstico imunológico). 0 principal tratamento indicado foram os antimoniais pentavalentes, porém, aqueles pacientes que entraram em protocolos de pesquisa recebiam os tratamentos de acordo às indicações dos ensaios. Na faixa etária de 0 a 5 anos, foram incluídas crianças até 71 meses de idade.

Foi construído um banco de dados no programa Fox-Pro 2,6 (Microsoft Corporation), utilizando como fonte secundária todas as fichas clínicas do CSCP, computando-se informações retrospectivas desde o mês de maio de 1987 (ano do início do funcionamento do CSCP), até dezembro de 1995.

A consistência e análise estatística dos dados foram feitas com o programa estatístico para Saúde Pública Epi Info 6 versão 6,03. Para a análise das diferenças clínicas (variáveis qualitativas), foi aplicado o teste do qui quadrado de Pearson com significância menor ou igual a 0,05 e calculando o OR (odds ratio) com intervalo de confiança 95\%. Para as variáveis quantitativas, foram calculados a média e o desvio padrão (DP), sendo comparados com o teste $t$, e análise de variância. Foram selecionados para a análise estatística os pacientes com diagnóstico de leishmaniose cutânea, mucosa e cutâneo mucosa. 0 estudo teve a aprovação do Comitê de Ética em Pesquisa em Seres Humanos da Universidade de Brasília.

\section{RESULTADOS}

Foram avaliadas 4.464 fichas clínicas arquivadas no CSCP que representam $98 \%$ do número total de atendimentos no
Centro entre maio de 1987 a dezembro de 1995. Do total de fichas revisadas, $4.432(99,3 \%)$ correspondiam a pacientes que apresentavam no momento da primeira consulta, ou durante a evolução, lesões confirmadas ou compatíveis com as diferentes formas clínicas de leishmaniose: cutânea (4.242 pacientes), mucosa (154 pacientes) ou cutâneo-mucosa ativa (36 pacientes). Foram avaliados os dados de 4.275 indivíduos cuja idade foi registrada nas fichas, dos quais, $491(11,8 \%)$ eram crianças de 0 a 5 anos de idade. A média anual geral de casos novos atendidos no CSCP, no período de 1987-1995, foi 492 pacientes e a média anual de casos em crianças de 0 a 5 anos foi 55, correspondendo a $11,2 \%$ do total de casos registrados.

$\mathrm{Na}$ análise por grupo etário e gênero, observou-se que no grupo de estudo, a proporção entre o gênero masculino e feminino foi 1,1:1, enquanto em maiores de 15 anos, a proporção masculino/feminino foi $2: 1$. Esta peculiaridade da população infantil não se observa quando se faz uma análise global, incluindo todos os grupos etários (Figura 1), onde a proporção total masculino/feminino mantém-se em aproximadamente 1,8:1. A forma clínica predominante neste grupo de crianças foi a cutânea $(98,4 \%)$, e nos maiores de 15 anos representou $94,1 \%$, sendo significativamente diferente $\left(X^{2}=15,66 \mathrm{p}=0,000\right)$. A forma mucosa e cutâneomucosa foi observada raramente $(1,6 \%)$ nestas crianças, diferente dos doentes com mais de 15 anos, onde a percentagem foi de $5,9 \%$.

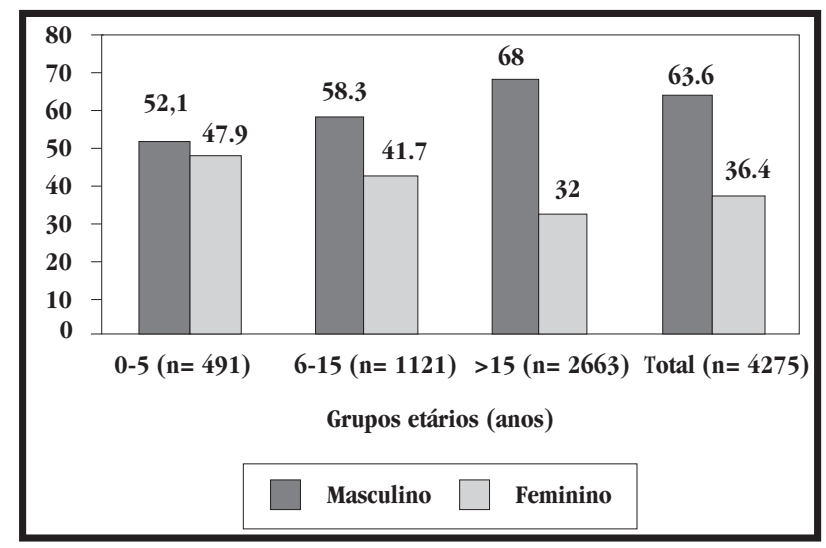

Figura 1 - Distribuição percentual segundo grupo etário e gênero de 4.275 pacientes com leishmaniose tegumentar atendidos no Centro de Saúde de Corte de Pedra - Bahia, Brasil. 1987 - 1995.

Comparou-se a distribuição corporal do total das lesões cutâneas entre a população de estudo e as de outras faixas etárias. $\mathrm{Na}$ população maior de 5 anos, a distribuição das lesões foi mais freqüente abaixo da cintura $(62,8 \%)$, e nas crianças predominaram acima da cintura $(63,5 \%)$, como mostra a Figura 2 $\left(\chi^{2}=155,9 \mathrm{p}<0,000\right)$. A chance de apresentar uma lesão localizada na parte superior do corpo foi três vezes maior para as crianças entre 0 a 5 anos quando comparada com os pacientes maiores de 15 anos (OR=3,2 IC95\% 2,6 a 3,8).

Quanto ao número de lesões por paciente, houve diferença significativa entre os grupos etários (Tabela 1). Nas crianças houve maior (41,2\%) número de lesões múltiplas (duas ou mais), comparado com os outros grupos etários: 6-15 anos (35,6\%) e, maiores de 15 anos (31,6\%), 


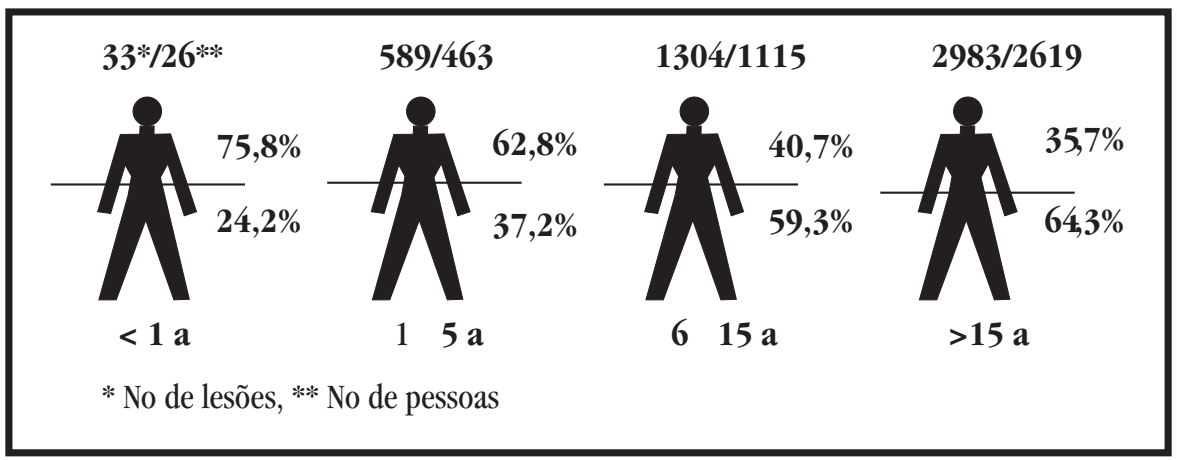

Figura 2 - Distribuição percentual das lesões cutâneas segundo a localização e grupo etário. Corte de Pedra - Bahia, Brasil. 1987 - 1995.

Tabela 1 - Distribuição percentual do número de lesões de leishmaniose tegumentar de acordo com o grupo etário. Corte de Pedra - Bahia. 1987-1995.

\begin{tabular}{lcccc}
\hline Número de lesões & \multicolumn{4}{c}{ Grupo etário } \\
\cline { 2 - 5 } & $\begin{array}{c}0-5 \text { anos } \\
\left(\mathrm{n}^{0}=490\right)\end{array}$ & $\begin{array}{c}6-15 \text { anos } \\
\left(\mathrm{n}^{0}=1.119\right)\end{array}$ & $\begin{array}{c}>15 \text { anos } \\
\left(\mathrm{n}^{0}=2.646\right)\end{array}$ & $\begin{array}{c}\text { Total } \\
\left(\mathrm{n}^{0}=4.255\right)^{*}\end{array}$ \\
\hline 1 & 58,8 & 64,4 & 68,4 & 66,2 \\
$2-3$ & 34,1 & 26,5 & 23,2 & 25,3 \\
$4-5$ & 4,9 & 6,4 & 3,9 & 4,7 \\
$>5$ & 2,2 & 2,7 & 4,6 & 3,8 \\
\hline
\end{tabular}

*Não foram analisados 20 pacientes cujo número de lesões não foi registrado nas fichas clínicas e três pacientes cuja idade não foi registrada

respectivamente $\left(\chi^{2}=19,19 \mathrm{p}<0,001\right)$. A média do número de lesões por paciente foi $1,7(\mathrm{DP}=1,9$; intervalo $=1 \mathrm{a} 48)$, sendo que nesta análise foram excluídos os pacientes com diagnóstico de leishmaniose disseminada ${ }^{7}$. Na distribuição das lesões nos diferentes grupos etários, observou-se para crianças entre 0 a 5 anos, média de 1,8 $(\mathrm{DP}=1,4$ intervalo $=1$ a 14$)$; entre 6 a 15 anos 1,8 (DP=1,7 intervalo $=1$ a 33$)$, e para os maiores de 15 anos $1,7(\mathrm{DP}=2,1$ intervalo $=1 \mathrm{a}$ 48). 0 tipo de lesão cutânea predominante na população geral atendida no CSCP foi a ulcerada $(99,3 \%)$, percentagem que variou pouco entre as diferentes faixas etárias, 99,6\% de 0 a 5 anos, 99,5\% de 6 a 15 anos e 99,3\% nos maiores de 15 anos.

A distribuição percentual do tempo de evolução das lesões, segundo a faixa etária, se apresenta na Tabela 2. Não houve diferença significativa entre o grupo de crianças e adolescentes para tempo de evolução $\leq 1$ mês, contudo houve uma diferença significativa entre as faixas etárias de 0 a 15 anos e maior de 15 anos $\left(\chi^{2}=6,17 \mathrm{p}=0,012\right)$. A prova $\mathrm{da}$ leishmanina foi realizada em 2.648 pacientes, e o resultado

Tabela 2 - Distribuição percentual do tempo de evolução das lesões cutâneas de leishmaniose de acordo com o grupo etário. Corte de Pedra, Bahia. 1987-1995.

\begin{tabular}{lcccc}
\hline $\begin{array}{l}\text { Tempo de evolução } \\
\text { (meses) }\end{array}$ & $\begin{array}{c}\text { G-5 anos } \\
\left(\mathrm{n}^{0}=488\right)\end{array}$ & $\begin{array}{c}6-15 \text { anos } \\
\left(\mathrm{n}^{0}=1.115\right)\end{array}$ & $\begin{array}{c}>15 \text { anos } \\
\left(\mathrm{n}^{0}=2.647\right)\end{array}$ & $\begin{array}{c}\text { Total* } \\
\left(\mathrm{n}^{0}=4.250\right)\end{array}$ \\
\hline$\leq 1$ & 53,3 & 54,3 & 50,1 & 51,6 \\
$2-3$ & 32,6 & 36,1 & 35,9 & 35,5 \\
$4-5$ & 8,0 & 5,8 & 7,0 & 6,8 \\
$>5$ & 6,1 & 3,8 & 7,1 & 6,1 \\
\hline
\end{tabular}

*Não foram analisados 25 pacientes cujo tempo de evolução não foi registrado e três pacientes cuja idade não foi registrada. foi registrado em 2.608. A Tabela 3 mostra os resultados segundo o tamanho da reação e a faixa etária. Em crianças de 0 a 5 anos, o percentual de positividade à prova da leishmanina $(93,6 \%)$, foi significativamente maior $\left(\chi^{2}=22,36\right.$ $\mathrm{p}<0,001)$ do que em crianças de 6 a 15 anos $(84,5 \%)$ e em maiores de 15 anos $(90,3 \%)$.

Tabela 3 - Distribuição percentual do resultado da prova da leishmanina em pacientes com leishmaniose cutânea segundo o grupo etário. Corte de Pedra, Bahia. 1987 -1995.

\begin{tabular}{lcccc}
\hline & \multicolumn{4}{c}{ Grupos etários } \\
\cline { 2 - 5 } Prova da leishmanina & $\begin{array}{c}0-5 \text { anos } \\
\left(\mathrm{n}^{0}=281\right)\end{array}$ & $\begin{array}{c}6-15 \text { anos } \\
\left(\mathrm{n}^{0}=631\right)\end{array}$ & $\begin{array}{c}>15 \text { anos } \\
\left(\mathrm{n}^{0}=1.696\right)\end{array}$ & $\begin{array}{c}\text { Total } \\
\left(\mathrm{n}^{0}=2.608\right)\end{array}$ \\
\hline Negativa & 6,4 & 15,5 & 9,7 & 10,8 \\
$5-10 \mathrm{~mm}$ & 49,5 & 39,1 & 45,0 & 44,1 \\
$11-20 \mathrm{~mm}$ & 39,1 & 33,0 & 37,9 & 36,8 \\
$>20 \mathrm{~mm}$ & 5,0 & 12,4 & 7,4 & 8,4 \\
\hline
\end{tabular}

\section{DISCUSSÃO}

A relação masculino: feminino encontrada na região de Corte de Pedra (BA) em crianças de 0 a 5 anos com IT foi semelhante à encontrada por outros pesquisadores no Brasil, Peru e na Bolívia, embora as faixas etárias estudadas tenham variado de 1 a 13 anos, 0 a 14 anos e 0 a 10 anos, respectivamente $e^{21026}$. Outros pesquisadores relataram relações maiores em faixas etárias que consideram até os 15 anos de idade ${ }^{2527}$. A equivalência encontrada quanto à distribuição por gênero aponta para a possibilidade de que crianças de ambos os gêneros permaneçam mais tempo no peridomicilio ou dentro de casa e estejam uniformemente expostas ao risco de transmissão nesses ambientes.

A predominância das lesões é cutâneas é semelhante aos casos relatados em São Paulo e na Argentina ${ }^{1021}$. Entretanto, Vallejo e Villalonga ${ }^{27}$, estudando crianças hospitalizadas em Tucuman-Argentina, relataram $27,4 \%$ de pacientes com lesão mucosa, percentagem bastante elevada quando comparada aos resultados em Corte de Pedra, onde se verificou apenas oito $(1,6 \%)$ casos com comprometimento mucoso, achado relacionado, provavelmente, ao atendimento hospitalar, aonde acorrem casos mais complicados.

Em Corte de Pedra, as lesões cutâneas se localizaram, preferencialmente, na parte superior do corpo, diferente de outras observações ${ }^{81}$ onde a localização predominante foi 
nos membros inferiores. Contudo, as faixas etárias estratificadas nestes trabalhos envolveram tanto crianças e adolescentes, os quais apresentam diferentes fatores de risco para adquirir a infecção.

A predominância de lesões cutâneas nos segmentos corporais superiores, como foi observado em Corte de Pedra, é semelhante aos achados em outras áreas de leishmaniose do Brasil ${ }^{10151722} \mathrm{e}$ nas Américas ${ }^{212} 2628$. A localização das lesões acima da cintura pélvica em crianças sugere um padrão de transmissão provavelmente intra ou peridomiciliar, porque existe coincidência do horário de maior atividade endofágica dos flebotomíneos com o momento em que as crianças estão no leito, com maior exposição da parte superior do corpo. Os pacientes com lesões localizadas acima da cintura teriam um risco maior de desenvolver lesões mucosas ${ }^{18}$, fazendo-se assim necessário o acompanhamento a longo prazo para identificar precocemente 0 aparecimento de lesões mucosas nas crianças de Corte de Pedra.

As lesões ulceradas foram as mais frequientes, semelhantes a outros estudos feitos na mesma área ${ }^{8}$, no Brasil ${ }^{22}$, e em outros países americanos ${ }^{21}$. Houve maior percentagem de lesões múltiplas, concordando com os achados de outros pesquisadores embora estes tenham estratificado faixas etárias diferentes ${ }^{810} 19$. Follador cols ${ }^{15}$ não encontraram diferença na proporção de crianças com lesões múltiplas, quando comparadas com os adultos, embora estes pesquisadores não mencionem a faixa etária das crianças estudadas.

Marsden ${ }^{20}$ levantou a possibilidade de que a existência de duas ou mais lesões no mesmo segmento do corpo seria o resultado de múltiplas picadas infectantes, referindo-se aos adultos, onde cada segmento corporal possui área maior, quando comparada à área dos mesmos segmentos em crianças. Assim sendo, as picadas infectantes poderiam atingir facilmente vários segmentos corporais de crianças em faixas etárias menores, o que explicaria a múltipla apresentação de lesões nesta faixa etária, sem haver necessariamente disseminação hematogênica para os explicar. Uma maior percentagem de lesões múltiplas favoreceria 0 aparecimento de lesões mucosas nas crianças ${ }^{18}$.

0 elevado percentual de positividade à prova da leishmanina, significativamente maior, que os outros grupos etários, é semelhante aos achados de outros pesquisadores ${ }^{810}$.

Neste trabalho, os achados sugerem que a leishmaniose em crianças na área de Corte de Pedra apresenta um padrão de transmissão intra ou peridomiciliar diferente do padrão apresentado em adolescentes e adultos, já descrito em outras áreas do Brasil, especialmente em surtos, quando atinge igualmente as diferentes faixas de idade ${ }^{15}$. Estes resultados poderão influenciar uma re-orientação das medidas de controle visando este grupo de idade empregando mosquiteiros ou cortinas impregnadas com inseticidas, ou a borrifação do domicílio, como profilaxia da transmissão da LT nesta faixa etária ${ }^{1613}$.

\section{AGRADECIMENTOS}

Ao Dr Gustavo Romero, pela revisão crítica do conteúdo deste manuscrito, ao Dr. João Barberino Santos pela correção ortográfica e sugestões na redação do texto e a Ednaldo Lima do Lago, pelo auxilio na identificação das fichas clínicas.

\section{REFERÊNCIAS BIBLIOGRÁFICAS}

1. Ashford RW. The leishmaniases as emerging and reemerging zoonoses. International Journal for Parasitology 30: 1269-81, 2000.

2. Bara MP. Estudio clínico epidemiológico de leishmaniasis cutánea en población pediátrica. Tesis de graduación. Universidad Peruana Cayetano Heredia. Lima, Perú, 1987.

3. Barrera C, Herrera M, Martinez F, León R, Richard A, Guderian RH, Mouchet J, Echeverria R, Le Pont F. Leishmaniose en Equateur. 1. Incidence de la leishmaniose tegumentaire sur la facade pacifique. Annales de la Société Belge de Médicine Tropicale 74: 1-12, 1994.

4. Bittencourt AL, Barral A, Costa JM. Tegumentary leishmaniasis in childhood. Pediatric Dermatology 13: 455-463, 1996.

5. Calmet J. Risk factors and leishmaniasis: possible contributions for control strategies. In: Leishmaniasis Control Strategies: A critical evaluation of IDRC-supported research, Ottawa p.206-222, 1992.

6. Campbell-Lendrum D, Dujardin JP, Martinez E, Feliciangeli MD, Perez JE, Silans LN, Desjeux P. Domestic and peridomestic transmission of American cutaneous leishmaniasis: changing epidemiological patterns present new control opportunities. Memórias do Instituto Oswaldo Cruz 96: 159-162, 2001.

7. Carvalho EM, Barral A, Costa JM, Bittencourt A, Marsden P. Clinical and immunopathological aspects of disseminated cutaneous leishmaniasis. Acta Tropica 56: 315-325, 1994.

8. Costa JM, Balby IT, Rocha EJ, da Silva AR, Rebêlo JM, Ferreira LA, Gama ME, Branco MR, Burattini MN, Soares NJ. Estudo comparativo da leishmaniose tegumentar americana em crianças e adolescentes procedentes das areas endemicas de Buriticupu (Maranhão) e Corte de Pedra (Bahia), Brasil. Revista da Sociedade Brasileira de Medicina Tropical 31: 279-288, 1998.

9. Costa JM, Vale KC, Cecilio IN, Osaki NK, Netto EM, Tada MS, França F, Barreto MC, Marsden PD. Aspectos psicossociais e estigmatizantes da leishmaniose cutâneo-mucosa. Revista da Sociedade Brasileira de Medicina Tropical 20: 77-81, 1987.

10. Cucé LC, Belda Jr W, Zolli CA. Leishmaniose tegumentar americana na infância. Aspectos epidemiológicos, clínicos e terapêuticos. Anais Brasileiros de Dermatologia 65: 18S-19S, 1990.

11. David C, Dimier-David L, Vargas F, Torrez M, Dedet JP. Fifteen years of cutaneous and mucocutaneous leishmaniasis in Bolivia: a retrospective study. Transactions of the Royal Society of Tropical Medicine and Hygiene 87: 7-9, 1993

12. Davies CR, Reithinger R, Campbell-Lendrum D, Feliciangeli D, Borges R, Rodriguez N. The epidemiology and control of leishmaniasis in Andean countries. Cadernos de Saúde Pública 16: 925-950, 2000.

13. Desjeux $P$. The increase in risk factors for leishmaniasis worldwide. Transactions of the Royal Society of Tropical Medicine and Hygiene 95: 239-243, 2001

14. Diógenes MJN, Goncalves H, Lima JWG, Menezes RHO. Leishmanioses na infância: aspectos epidemiológicos, clínicos e terapêuticos (Estado do Ceará-Brasil). Anais Brasileiros de Dermatologia 68: 209-212, 1993.

15. Follador I, Araujo C, Cardoso MA, Tavares-Neto J, Barral A, Miranda JC, Bittencourt A, Carvalho EM. Surto de leishmaniose tegumentar americana em Canoa, Santo Amaro, Bahia, Brasil. Revista da Sociedade Brasileira de Medicina Tropical 32: 497-503, 1999.

16. França F, Lago EL, Tada S, Costa JM, Vale K, Oliveira J, Costa MA, Osaki M, Cheever L, Netto EM, Barreto AC, Johnson WD, Marsden PD. An outbreak 
of human Leishmania (Viannia) braziliensis infection. Memórias do Instituto Oswaldo Cruz 86: 169-174, 1991.

17. Hermeto MV, Vieira-Dias D, Genaro O, Rotondo-Silva A, da Costa CA, Toledo VP, Michalick MS, Williams P, Mayrink W. Outbreak of cutaneous leishmaniasis in the Rio Doce Valley, Minas Gerais, Brazil. Memórias do Instituto Oswaldo Cruz 89: 519-521, 1994.

18. Llanos-Cuentas EA, Marsden PD, Cuba CC, Barreto AC, Campos M. Possible risk factors in development of mucosal lesions in leishmaniasis. Lancet 2: 295, 1984.

19. Llanos-Cuentas EA, Marsden PD, Lago EL, Barreto AC, Cuba CC, Johnson WD. Human mucocutaneous leishmaniasis in Três Bracos, Bahia - Brazil. An area of Leishmania braziliensis braziliensis transmission. II. Cutaneous disease. Presentation and evolution. Revista da Sociedade Brasileira de Medicina Tropical 17: 169-177, 1984

20. Marsden PD. Clinical presentations of Leishmania braziliensis braziliensis. Parasitology Today 1: 129-133, 1985.

21. Miranda 0, Gimenez MF, Waisman R, Gimenez MM. Leishmaniasis infantojuvenil. Centro Dermatológico - Resistencia - (1975-1986). Revista Argentina de Dermatologia 69: 91-95, 1988.

22. Nishino L, Ragiotto AR, Almeida NA, Santamaria JR. Leishmaniose em criança. Anais Brasileiros de Dermatologia 61: 23-28, 1986.
23. Romero GA, Vinitius De Farias Guerra M, Gomes Paes M, Macêdo Vo. Comparison of cutaneous leishmaniasis due to Leishmania (Viannia) braziliensis and $L$. (V.) guyanensis in Brazil: clinical findings and diagnostic approach. Clinical Infectious Diseases 32: 1304-1312, 2001

24. Rosa AC, Cuba CC, Vexenat A, Barreto AC, Marsden PD. Predominance of Leishmania braziliensis braziliensis in the regions of Três Braços and Corte de Pedra, Bahia, Brazil. Transactions of the Royal Society of Tropical Medicine and Hygiene 82: 409-410, 1988.

25. Scorza JV, Valera M, Moreno E, Jaimes R. Encuesta epidemiológica sobre leishmaniasis cutánea. Un estudio en Mérida, Venezuela. Boletín de la Oficina Sanitaria Panamericana 95: 118-133, 1983.

26. Torres Espejo JM, Le Pont F, Mouchet J, Desjeux P, Richard A. Epidemiologie de la leishmaniose tegumentaire en Bolivie. 1. Description des zones d'etude et frequence de la maladie. Annales de la Société Belge de Médicine Tropicale 69: 297-306, 1989.

27. Vallejo L, Villalonga J. Leishmaniasis infantil. Revista Argentina de Dermatologia 66: 1-7, 1985.

28. Velez ID, Hendrickx E, Robledo SM, Agudelo SP. Leishmaniosis cutánea en Colombia y género. Cadernos de Saúde Pública 17: 171-180, 2001. 